\title{
Define the early biomarker
}

\section{Opinion}

How early is early? Early enough to prevent or early enough to treat? For different conditions, early was defined differently often by symptoms and signs.

Can we have a general definition of early biomarker? In terms of biomarker discovery, I personally prefer to cut between the homeostasis and loss of homeostasis in blood. ${ }^{1}$ If the blood remain homeostatic, no doubt, it is in early phase. You can even say it is not in diseased state yet at all. But the damage is done already. A normal blood glucose level does not mean a healthy metabolic condition. A normal blood creatinine level does not mean a healthy kidney. Diseases develop before they even be called diseases. The homeostatic mechanisms of the body are working hard to keep the blood stay in the normal range. The early phase is the period when we can and should start to intervene to stop the disease process. We have not done enough of it yet, because we do not know the early phase well enough. We cannot tell when it is the early phase. Why can't we tell the early phase? Because for most biomarker studies, the concentration was put too much on finding biomarkers in blood. From the definition above, we can see that the early biomarkers are not even in the blood, the blood is not changed yet because of the homeostatic mechanisms are still working well enough to keep the blood in the normal range.

How can we find the early biomarker? We have to look at the place where the wastes are dumped while the homeostasis mechanisms work. Those are the most sensitive places to observe the early changes associated with the early disease process. I personally prefer to recommend urine at current stage even breath, sweat, mucous secretions, bile should not be ignored. One of the most important advantages of urine as a biomarker source has been urine can be obtained noninvasively compare to blood. This advantage is too subtle. Another advantage was urine can be obtained in large quantity. This is probably easily offset by its dilution. The real biggest advantage of urine as biomarker source is that urine has more changes
Volume 3 Issue 2 - 2016

\section{Youhe Gao \\ Department of Biochemistry and Molecular Biology, Beijing Normal University, China}

Correspondence: Youhe Gao, Department of Biochemistry and Molecular Biology, Beijing Normal University, Gene Engineering and Biotechnology Beijing Key Laboratory, Beijing, I00875, PR. of China, Tel 86I058804382,

Email gaoyouhe@bnu.edu

Received: January 25, 2016 | Published: February 05, 2016

in early phase. In other words, urine has early biomarkers when blood is still normal. ${ }^{2,3}$ Defining early biomarker now is to call the attention of biomarker researchers to think if we have been looking for the early biomarker in a wrong place.

\section{Acknowledgements}

This work was supported by the National Basic Research Program of China (2012CB517606, 2013CB530805), the Key Basic Research Program of China (No. 2013FY114100).

\section{Conflict of interest}

The author declares no conflict of interest.

\section{References}

1. h t t p : / / b log. s c i e n c e n e t. c n / ho m e. php? mod $=$ space $\&$ uid $=244733 \&$ do $=$ blog $\&$ id $=951332$

2. Gao Y. Urine-an untapped goldmine for biomarker discovery? Sci China Life Sci. 2013;56(12):1145-1146.

3. Wu J, Gao Y. Physiological conditions can be reflected in human urine proteome and metabolome. Expert Rev Proteomics. 2015;12(6):623-636. 